\title{
APPLICATION OF BEARING AND DISTANCE TREES TO THE IDENTIFICATION OF LANDMARKS ON THE COAST
}

\author{
TOMASZ PRACZYK
}

\author{
Institute of Navigation and Hydrography, Naval University of Gdynia \\ ul. Śmidowicza 69, 81-103 Gdynia, Poland \\ e-mail: T.Praczyk@amw.gdynia.pl
}

\begin{abstract}
The problem of continuous position availability is one of the most important issues connected with the human activity at sea. Because the availability of satellite navigational systems can be limited in some cases, e.g. during military operations, one has to consider additional methods of acquiring information about the ship's position. In this paper one of these methods is presented, which is based on exploiting landmarks located on a coastline. A navigational radar is used to obtain information about these points. In order to estimate the ship's position by means of a set of landmarks, it is necessary to know their accurate locations. The paper presents a landmark identification method based on the comparison of bearing and distance trees representing pattern points generated from a chart, as well as points extracted from a radar image.
\end{abstract}

Keywords: ship's positioning, landmark identification, bearing and distance tree

\section{Introduction}

A primary positioning system can be turned off, destroyed or malfunctioning. This is the reason why alternative methods of position estimation should be taken into account. It is especially important for naval ships. A military, auxiliary positioning system should be as autonomous as possible. It should offer a possibility to estimate the ship's position without any help from outside. Another crucial issue concerning military positioning systems is robustness against external disturbances. The more the opponent has to do in order to distort navigational information, the better the system is. The possibility to estimate the position on any stretch, i.e. the global character of the navigational system, is its last important feature. A navy ship should be capable of executing a task wherever it is located and its navigational system should make it possible. Summarizing, we can enumerate four key properties of military spare positioning systems: accuracy, autonomy, robustness and the global character.

In the article, solutions are presented which are autonomous, robust to external disturbances, relatively accurate, but not global. They make it possible to estimate the position solely in coastal stretches. This limited applicability results from using a navigational radar as the main source of information in the process of position estimation. All bigger sea going vessels are equipped with such radars and their application in navigation is not a new idea. A traditional method of radar positioning makes use of landmarks located on the coast, which are present in the sea area and visible on a radar image. Knowing the accurate position of a reference point along with its bearing and the distance to it, we are able to estimate the ship's position. Unfortunately, the identification of a point appearing in the image, i.e. assigning it a counterpart on a chart, constitutes a frequent problem for navigators. For that reason, other methods are used. The first of them is the so-called comparative navigation (Stateczny, 2001). It assumes that each position (near the coast) corresponds to a characteristic radar image of the coastline. In short, comparative positioning consists in constructing a database of pattern radar images (from known positions) and using all memorized information together with simple minimal distance algorithms during the positioning process: the pattern image closest to the image recorded from the navigational radar corresponds to the ship's position.

This approach possesses three major drawbacks. The first is related to difficulties with obtaining appropriate training data. The most natural but, at the same time, the most expensive solution is registering real radar images from the coast area of interest. In addition to the huge costs entailed, we will never have a guarantee that the data obtained in such a way will represent everything we can 
get on the radar screen. In order for such data to become a reliable base on which we could estimate the position, they should be registered in various weather conditions, in different seasons, at night, in daytime, etc. All factors that could have influence on the radar image should be taken into account. The resulting costs would thus be very high.

Except difficulties with acquiring reliable training data, the solution based on real radar images has one more disadvantage. Namely, in order to create a positioning system which would be useful practically on any coastal stretch, we would have to have at our disposal radar images coming from all possible coastal areas. Moreover, as it has been mentioned before, training images should take into consideration various factors that could influence the radar image. Acquiring such data is practically impossible and, therefore, the application of the system prepared based on real radar images is limited to known nearly areas of the sea.

Another possibility to obtain training data is to simulate them from a paper or electronic chart. It is a cheap solution since it does not demand a registration in real conditions. Furthermore, it makes the creation of a global positioning system possible because we can generate training data coming from any stretch. Unfortunately, the differences between radar images and the corresponding images generated from a chart hinder the application of the latter images in comparative navigation positioning systems. The differences concern both the coastline and remote elements of the land that are very often not visible on the radar image.

The second drawback of traditional comparative navigation is its poor accuracy. It results from the idea of positioning applied. Answers (positions) of the system are restricted only to positions corresponding to memorized pattern images. In order to estimate the position between two training images we have to add the next image.

The last shortcoming of comparative navigation is its sluggishness. In order to estimate the position, the system has to compare a registered image with the whole set of pattern images.

A newer approach to the problem of positioning at sea is to use for that purpose artificial intelligence methods such as artificial neural networks (Praczyk, 2006b). They may constitute a reasoning element whose task is to assign the position to the radar image registered. In this case, except difficulties in obtaining appropriate training data, there is a problem with their dimensionality. The most important criterion for estimation by a positioning system is its accuracy. If we wish to obtain a precise positioning system, it is necessary to collect a large number of radar images (or simulated radar images) from the coastal area that we are interested in. This usually means hundreds and even thousands of images, a couple of images per position. This is a massive amount of data, which implies that we are faced with an extremely complex task. Training a neural network based on such massive data may become practically impossible and at least very troublesome and time consuming.

A way out is the application of different methods which simplify the problem complexity. On the one hand, a decomposition into simpler problems can be used in which the sea area is partitioned into smaller regions, each with its own positioning system. Another method of reducing the complexity is the application of radar image feature extraction methods (leading to image compression) (Praczyk, 2006d). The information contained in the radar image is reduced to an amount acceptable from the point of view of further computations.

Feature extraction methods are not used solely as a panacea for the acceleration of computations in the inference or training phases. Very often their application is a necessary condition for proper functioning of the positioning system. Such a situation happens when training images are generated from an electronic or paper chart. In such a case accurate positioning without preprocessing input data and without the fusion of all those data is impossible. Feature extraction methods gather from images only information which is essential for the task, while omitting all that is unnecessary or even detrimental.

Unfortunately, as prior investigations the exhibited (Praczyk, 2006b), it is extremely difficult to construct a sufficiently accurate positioning system which would work based on a neural network and a compressed radar image. There are two possible reasons behind this situation. The first is the fact that a position function defined on a feature vector domain can be strongly nonlinear, making its approximation very difficult. The nonlinearity of the position function results, in turn, from a dispersion of radar image equivalents, which are originally located close to one another. In a radar image domain, images corresponding to close positions are similar. In turn, images representing distant positions are usually extremely different. Unfortunately, the feature extraction process can destroy concentrations occurring in the radar image domain scattering radar images representatives in different places of the feature vector domain. Neighbouring vectors may correspond to extremely distant positions. The greater the level of dispersion, the more difficulties in estimating the accurate position.

Another important reason for the difficulties observed while creating a valuable positioning system working based on compressed radar images and neural networks is the fact that, apart from information essential for the positioning process, every radar image also contains noise. What is more, this noise may constitute a large fragment of the image distorting it significantly. Of course, there are methods which make it possible to extract from the image the least deformed element, namely, the coastline. However, the coastline visible on the radar image may also differ depending on weather, season, etc. 
Consequently, the position approximated based on an even slightly deformed coastline could be strongly inaccurate.

Another approach also taking advantage of a neural network as a position approximator estimates the position based not on the entire radar image or its feature vector descriptions, but on a vector of bearings and distances to a specially arranged system of buoys (Praczyk, 2006c). Distances and bearings to buoys can be obtained directly from the radar. In such a case, the neural network learns by means of a set of sample vectors of bearings and distances generated from a chart. In an inference phase, the network receiving from the radar the vector of bearing and distances to a set of properly ordered buoys (from the north-most buoy to the south-most buoy) is able to approximate the vessel's position.

This method has two major disadvantages. First, it is not global. Positioning is possible solely in areas equipped with a system of distinguishable buoys located in fixed positions. Thus, in order to estimate the position, the presence of buoys is not sufficient. Buoys should be characteristic or produce characteristic radar echoes and they should not change locations over time. Consequently, we may use the system in a specific coastal area and nowhere else.

The second disadvantage of the concept using bearings and distances to buoys in order to estimate the position is its large sensitivity to navigational information disturbances. For example, serious problems appear if we add an additional buoy imitating a buoy belonging to the system. What is worse, we will not know that the generated position is be erroneous.

The automation of traditional radar navigation is another positioning method (Praczyk, 2006a). In this case, positioning is based on characteristic points of the coast (ends of peninsulas, buoys, etc.), which constitute the most reliable elements of the image and, what is also important, the positioning is reduced to the identification of such landmarks and subsequent estimation of the position. At the beginning, landmarks are extracted from the radar image, then they are identified and, finally, the identified landmarks are used as reference points, i.e. the bearing and distance to each of them are determined, which suffices to estimate the ship's position. The method makes it possible to estimate the position on all coastal stretches where we can find landmarks. It does not require any expensive measurements at sea in order to construct the system. It only suffices to have knowledge that we can acquire from a navigational chart. The system malfunctioning is very difficult because every point that can constitute detrimental noise should be detected by the system and neglected in the process of position estimation.

The article presents the last of the presented positioning approaches, focusing particularly on a new landmark identification method. The paper begins with the presentation of a framework of the proposed positioning system in Section 2. Section 3 outlines the first element of the entire system, i.e. the landmark extraction subsystem. In Section 4, the proposed method of landmark identification is characterized. Experimental results are included in Section 5 and conclusions are made at the end of the paper.

\section{System Description}

The proposed positioning system consists of five main elements: a radar, a radar image registration subsystem, a landmark extraction subsystem, a landmark identification subsystem and a positioning subsystem. The structure of the whole system is presented in Fig. 1.

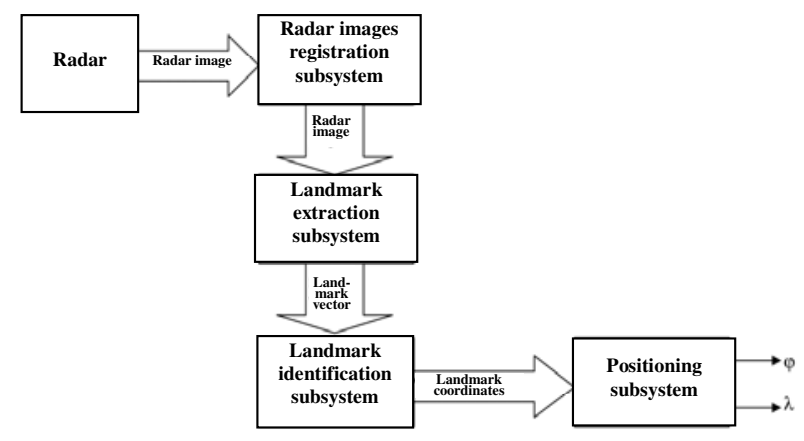

Fig. 1. Structure of the positioning system.

The role of the radar is evident and, therefore, we shall focus only on the remaining four elements. The main task of the radar image registration subsystem is transformation of the radar signal to a digital form. An additional task of this subsystem is the elimination of echoes coming from moving ships. In the image, they look like small points so they can be interpreted as spurious landmarks hindering the identification of the true landmarks. For that reason, if it is possible, they should be eliminated from the image. To this end, the subsystem should be able to track every object that changes its location over time.

The detection of such an object could be accomplished, e.g. through simple summation of images and the application of a threshold filter to the resulting image. It removes all points whose brightness is less than the assumed threshold. In two original images the echo of a moving object is in different places, so in the resulting image the echo of the object is visible as a short line. However, the brightness of this line is lower than that of the remaining stationary objects visible in the image. It follows that after the application of a threshold filter the line will be removed (Kuchariew, 1999).

The main task of the landmark extraction subsystem is the preparation of a vector of characteristic points visible in the registered radar image. The method of landmark extraction constitutes the subject of the next section. In the subsequent phase, the landmark identification follows-a position is assigned to each landmark. In the final step, 
the system estimates the ship's position using the identified landmarks and traditional radar navigational methods.

The entire system should work for various ranges of radar observation. The application of longer ranges implies that during reasoning we would use landmarks covering larger areas of the sea while neglecting points apparent only for shorter ranges. The application of longer ranges also entails that the estimates of bearings and distances necessary to identify the ship's position may be inaccurate. For that reason, after the initial landmark identification and a rough position determination, the system should be run for shorter ranges in order to consider more details present in the image, to verify the correctness of the previous decisions, but also to estimate landmark positions more accurately.

The system needs a database of pattern points from a chart. The points can be estimated either automatically by the landmark extraction subsystem or manually by the system operator by marking them on the chart. Each point should be described and assigned an accurate position. The pattern points should be both very marked points visible for large scales of the chart and less noticeable points observable in the radar image only for shorter ranges of the radar observation. In order to speed up the system, a radar observation range should be assigned to every pattern point, apart from a description and an accurate position. Thus, during identification for larger observation ranges solely a part of points, not all of them, would be taken into account. Less marked points apparent only for shorter ranges would be omitted. Furthermore, for shorter observation ranges the whole area considered should be partitioned into sub-areas. In this way, only points located near a rough position of the vessel estimated for a larger range would be taken into account.

\section{Landmark Extraction Subsystem}

As far as landmark extraction from the radar image is concerned, we should start from presenting the image considered in the form of a contour invariant. The contour invariant of an image is a notion introduced in (Kuchariew, 1999) and, regarding a radar image, it relates to the line of coast visible from the ship, presented in a polar coordinate system. We can define it as follows (Praczyk, 2006a):

$$
g_{\mathrm{inv}}(B R G)=\left\{\begin{array}{cl}
A & \text { if } P_{B R G, c}^{O}=\emptyset, \\
\min _{p \in P_{B R G, c}^{O}}\left|p_{c} p\right| & \text { otherwise }
\end{array}\right.
$$

where $B R G \in\left[0^{\circ}, 360^{\circ}\right)$ is the bearing from the central point of the image $p_{c}\left(n_{c}, m_{c}\right)\left(n_{c}=\frac{1}{2}(H-1), m_{c}=\right.$ $\frac{1}{2}(W-1)$ and we assume that $H$ and $W$ which determine the size of the image are odd), $P_{B R G, c}^{O} \subset P^{O}$ is a subset of the set of all radar image points that includes "bright" pixels lying on the bearing $B R G$ and $A$ is some value larger than the distance between the central point and any other point of the image. In order to simplify the landmark extraction algorithm, the function (1) is replaced by

$$
g_{\text {inv }}^{b}(k)=\left\{\begin{array}{cl}
A & \text { if } P_{B R G_{k}, c}^{O}=\emptyset, \\
\min _{p \in P_{B R G_{k}, c}^{O}}\left|p_{c} p\right| & \text { otherwise }
\end{array}\right.
$$

for $k=0,1, \ldots, 2(H+W)-4$, where $k$ is the index of a border point

$$
p_{b, k} \in\left\{(n, m) \in P^{O}: n=H \text { or } m=W\right\}
$$

in an ordered series of border points, and $B R G_{k}$ is the bearing at the $k$-th border point of the image.

A continuous equivalent of $g_{\text {inv }}^{b}(k)$, defined in

$$
C=\{x \in \mathbb{R}: 0 \leq x \leq 2(H+W)-4\},
$$

is $g_{\text {inv }}^{C}(x)$ such that $g_{\text {inv }}^{C}(k)=g_{\text {inv }}^{b}(k)$. We can treat $g_{\text {inv }}^{C}(x)$ as an analog radar image contour invariant. The proposed method of landmark extraction is based on the analysis of the second derivative of $g_{\mathrm{inv}}^{C}(x)$. We do not know $g_{\text {inv }}^{C}(x)$, but to estimate landmarks, we can employ its estimator $\hat{g}_{\text {inv }}^{C}(x)$ created based on the pairs $\left(k, g_{\text {inv }}^{b}(k)\right)$. As the estimator, we may use the following:

$$
\hat{g}_{\text {inv }}^{C}(x, \sigma)=\frac{\sum_{k=0}^{2(H+W)-5} g_{\text {inv }}^{b}(k) \varphi_{k}(x, \sigma)}{\sum_{k=0}^{2(H+W)-5} \varphi_{k}(x, \sigma)},
$$

where

$$
\varphi_{k}(x, \sigma)=\exp \left(-\frac{(x-k)^{2}}{2 \sigma^{2}}\right) .
$$

The algorithm consist in calculating

$$
z(x, \sigma)=\frac{\mathrm{d}^{2} \hat{g}_{\text {inv }}^{C}}{\mathrm{~d} x^{2}}
$$

for a fixed $\sigma$ at points $x=k=0,1, \ldots, 2(H+W)-4$, and then finding $k$ for which the conditions

$$
\begin{aligned}
& z(k)>\lambda, \quad z(k)>z(k-1), \\
& z(k) \geq z(k+1), \quad g_{\text {inv }}^{b}(k) \neq A
\end{aligned}
$$

hold, where $\lambda$ permits to partition the set of potential landmarks into two subsets: visible $(z(k)>\lambda)$ and hardly visible $(z(k) \leq \lambda)$. Another parameter of the algorithm is $\sigma$. We can say that $\lambda$ eliminates hardly visible points from the list of points while $\sigma$ prevents generating them.

Denote by $\Phi$ the subset of the indexes of the border image points that fulfil (5). Finally, the points

$$
(n, m)=f_{P c}\left(f_{\varphi d \rightarrow x y}\left(B R G_{k}, g_{\text {inv }}^{b}(k)\right)\right), k \in \Phi,
$$


are considered as landmarks in the radar image. $f_{\varphi d \rightarrow x y}$ is a conversion function between the polar coordinate system and the Cartesian coordinate system, whereas

$$
f_{P c}(q)=\arg \min _{p \in P^{O}}|p q|
$$

is a function which returns the radar image point which is closest to a given point $q(x, y), x, y \in \mathbb{R}$.

\section{Landmark Identification Subsystem}

After the landmarks have been extracted from the radar image, their identification is performed. It consists in assigning them the positions of certain pattern characteristic points which had been selected in a preparatory phase. In (Praczyk, 2006a), an identification method was presented which consists in the comparison of coastlines visible from pattern and radar image points. The coastlines for pattern points are generated from the image that is created from an electronic chart. Given a bearing $B R G$, the coastline visible from an image point $p$ is defined as follows:

$$
g^{p}(B R G)=\left\{\begin{array}{cl}
A & \text { if } P_{B R G, p}^{O}=\emptyset \\
\min _{q \in P_{B R G, p}^{O}}|p q| & \text { otherwise }
\end{array}\right.
$$

where $P_{B R G, p}^{O}$ is the set of "bright" pixels lying on the bearing $B R G$ estimated from the point $p$ and $A$ is some value larger than the distance between any two points of the image. According to (7), each coastline includes information where we can see dry land $\left(g^{p} \neq A\right)$ and where we cannot $\left(g^{p}=A\right)$.

During identification, two factors are considered: the similarity between the coastlines and the identification risk. The similarity is expressed as the normalized Euclidean distance between the coastlines. In turn, the identification risk of a given radar image point depends on the total length of the mainland visible from that point. The longer the segment of the visible dry land, the lower the identification risk. Radar image characteristic points that have appropriately similar counterparts among pattern points and their identification risk is suitably low are considered as reference points in the position estimation process.

This approach has two basic drawbacks. The least deformed element of a coast radar image is the coastline visible from the ship that is in the centre of the image. Fragments of the image located behind the coastline are most uncertain and making a decision based on them is very risky. The coastline visible from any point of the radar image other than the central point does not agree with the coastline appearing from the latter. The coastlines can partially include the same fragments of the land but they can also be segments indicating distinct areas of the radar image. Therefore, the representation of characteristic points of the radar image may include only a part of all reliable information that we can obtain from the image, while omitting obscured fragments of the coastline visible from the ship. What is more, it may also contain fragments that are rather detrimental for the landmark identification process.

The second drawback of the landmark identification method proposed in (Praczyk, 2006a) is the determination of the similarity between the coastlines. Consider the following situation: We have one pattern point and the corresponding coastline generated from a chart. We have also two characteristic points extracted from a radar image together with their coastlines. The first point is noisecorrupted, whereas the second point corresponds in reality to our pattern point, but it has a strongly deformed coastline in some fragment of the image (for some range of bearings). Most of the coastline is very similar to the pattern coastline, but there is some continuous segment which significantly differs from the pattern. We can encounter such a situation, e.g. in winter, when some areas of the sea can be covered by ice. Consequently, the similarities between the pattern coastline and the coastlines representing both points extracted from the radar image may be comparable and the point which should be correctly identified will be rejected by the identification subsystem.

The application of the information about bearings and distances between characteristic points of the coast is the identification method that is devoided of all the shortcomings presented above. This time the identification of a point is carried out based on the location of the remaining characteristic points with respect to the point we wish to identify. Therefore, the elements of the land visible from the ship are solely taken into consideration during identification. Moreover, local image deformations should not influence identification results. Noise-corrupted characteristic points that can be generated in a deformed fragment of the image differ significantly from pattern points so they are easy to recognize and eliminate by the identification system.

4.1. Mathematical Formulation. Let $P^{I} \subset P^{O}$ denote the set of all $L$ landmarks $p_{i}^{I}\left(n_{i}, m_{i}\right), i=1, \ldots, L$ generated from the radar image. The mutual location of any two radar image landmarks $p_{i}^{I}$ and $p_{j}^{I}$ is determined by the bearing $B R G_{i j}^{I}$ from $p_{i}^{I}$ to $p_{j}^{I}$ and the bearing $B R G_{j i}^{I}$ from $p_{j}^{I}$ to $p_{i}^{I}$, where $B R G_{i j}^{I}=360^{\circ}-B R G_{j i}^{I}$ and $d_{i j}^{I}=d_{j i}^{I}$ is the distance between the points. The above situation could be presented in the form of a directed graph $G^{I}$, in which the landmarks from $P^{I}$ constitute the vertices while the edges are described by bearings and distances. The graph $G^{I}$ can be defined by means of three matrices: $\boldsymbol{M}^{G^{I}}=\left[m_{i j}^{G^{I}}\right]_{L \times L}, \boldsymbol{D}^{G^{I}}=\left[d_{i j}^{G^{I}}\right]_{L \times L}$ and $\boldsymbol{B R} \boldsymbol{G}^{G^{I}}=\left[B R G_{i j}^{G^{I}}\right]_{L \times L} \cdot \boldsymbol{B} \boldsymbol{R} \boldsymbol{G}^{G^{I}}$ and $\boldsymbol{D}^{G^{I}}$ are respec- 


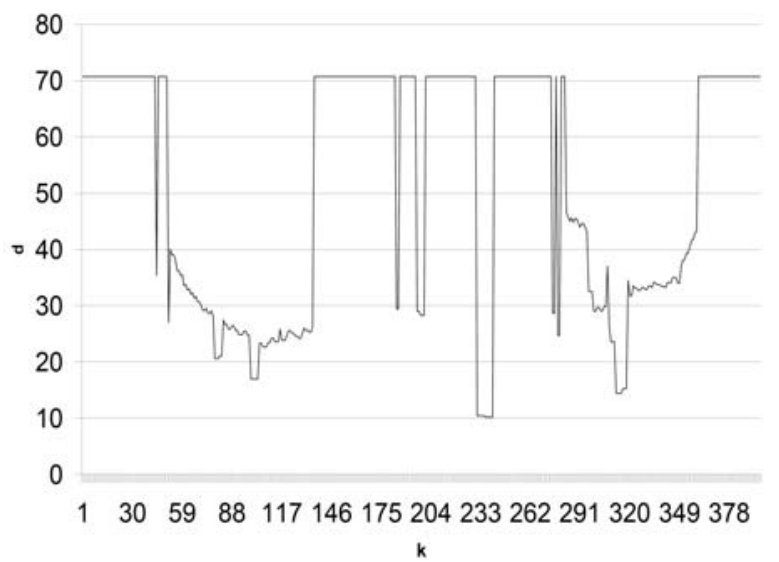

(a)

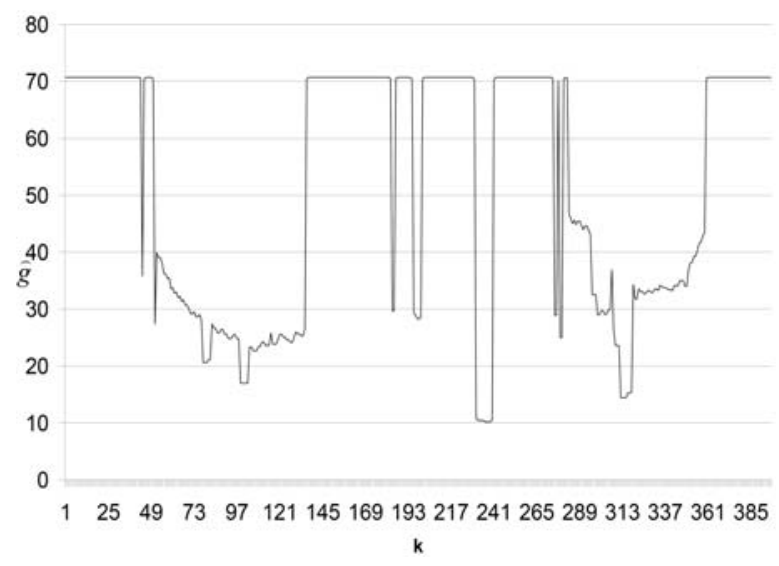

(b)

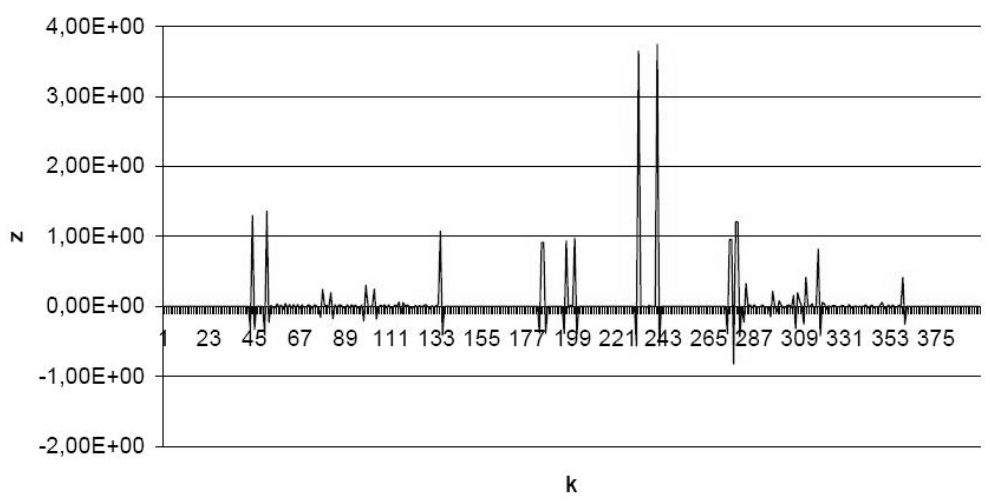

(c)

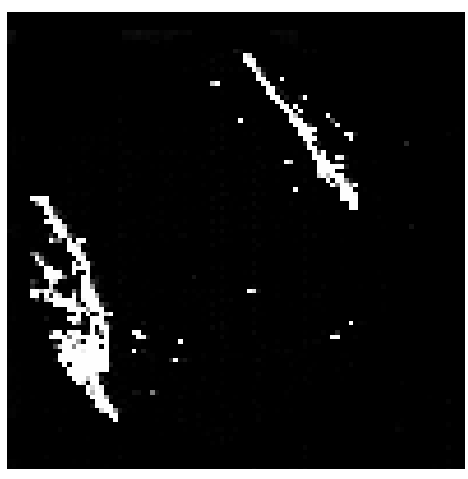

(d)

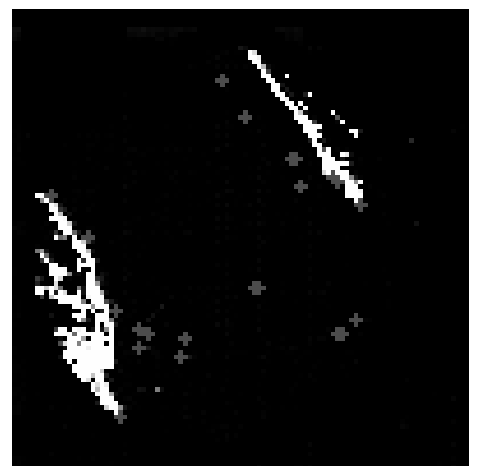

(e)

Fig. 2. (a) Contour invariant of a sample radar image, (b) $\hat{g}_{\text {inv }}^{C}$ for $\sigma=0.1$, (c) $z(k)$ for $\sigma=0.1$, (d) a sample radar image, (e) the same image with characteristic points estimated for $\sigma=0.1, \lambda=0.5$.

tively bearing and distance matrices. Each edge $g_{i j}^{G^{I}}$ of the graph $G^{I}$ that connects the $i$-th and $j$-th points is described by the $\operatorname{pair}\left(B R G_{i j}^{G^{I}}, d_{i j}^{G^{I}}\right) . M^{G^{I}}$ is a neighbourhood matrix of the graph $G^{I}$ that determines which connections between points occur and which do not. We have that for each $i, j=1, \ldots, L$,

$$
m_{i j}^{G^{I}}= \begin{cases}1 & \text { if } i \neq j \\ 0 & \text { otherwise. }\end{cases}
$$

Then, let $G^{I, i}$ be the tree describing the point $p_{i}^{I}$ created through removing from $G^{I}$ all edges not running from the point $p_{i}^{I}$. The neighbourhood matrix $M^{G^{I, i}}$ of $G^{I, i}$ could be defined as follows:

$$
m_{k j}^{G^{I, i}}= \begin{cases}1 & \text { if } k=i \text { and } k \neq j . \\ 0 & \text { otherwise }\end{cases}
$$

where $k, j=1, \ldots, L$. 
Subsequently, let $P^{W} \subset P^{G}$ be the set of the $K$ pattern landmarks $p_{i}^{W}\left(\varphi_{i}, \lambda_{i}\right), i=1, \ldots, K$ of the sea area considered. $\varphi_{i}$ and $\lambda_{i}$ determine the position of the $i$-th pattern landmark (latitude and longitude) and $P^{G}$ is the set of all possible points on earth in the geographical coordinate system. Let $G^{W}$ be a directed graph in which the vertices are the pattern landmarks and every vertex is connected with other vertices except itself through two reversely directed edges described by a bearing and a distance. The matrices $\boldsymbol{M}^{G^{W}}=\left[m_{i j}^{G^{W}}\right]_{K \times K}$, $\boldsymbol{D}^{G^{W}}=\left[d_{i j}^{G^{W}}\right]_{K \times K}$ (distance matrix) and $\boldsymbol{B} \boldsymbol{R} \boldsymbol{G}^{G^{W}}=$ $\left[B R G_{i j}^{G^{W}}\right]_{K \times K}$ (bearing matrix) complete the definition of $G^{W}$. We have that for each $i, j=1, \ldots, K$,

$$
m_{i j}^{G^{W}}= \begin{cases}1 & \text { if } i \neq j \\ 0 & \text { otherwise. }\end{cases}
$$

In much the same way as in the case of radar image landmarks, we can represent every pattern landmark in the form of a tree $G^{W, i}$ for which the neighbourhood matrix $\boldsymbol{M}^{G^{W, i}}$ could be defined as follows:

$$
m_{k j}^{G^{W, i}}= \begin{cases}1 & \text { if } k=i \text { and } k \neq j \\ 0 & \text { otherwise }\end{cases}
$$

where $k, j=1, \ldots, K$.

If we wish to compare radar image and pattern landmarks from a chart, we should bear in mind that from a given radar image landmark we can see only points whose distance to the radar image landmark considered is no greater than some value $\delta_{j}^{R}(B R G)$, where $j$ is the index of the analysed radar image point. This value is dependent on the radar observation range $R$ and the bearing $B R G$ estimated from the radar image landmark considered. Until now, the representation of every pattern landmark, i.e. the tree $G^{W, i}$ has not taken account of the radar's restricted field of vision. In order to make $G^{W, i}$ and $G^{I, j}$ describing the $i$-th pattern landmark and the $j$-th radar image landmark comparable, we should remove from $G^{W, i}$ every landmark that for a given bearing $B R G$ is located further from the $i$-th pattern landmark than $\delta_{j}^{R}(B R G)$.

Denote by $G_{R}^{W, i, j}$ this new tree, where $i$ and $j$ are the indices of the landmark and the radar image landmark, respectively. The elements of the neighbourhood matrix $M^{G R}$ could be defined as follows:

$$
m_{k l}^{G R}=\left\{\begin{array}{cc}
1 & \text { if } k=i \text { and } k \neq l \\
& \text { and } d_{k l}^{G W} \leq \delta_{j}^{G}\left(B R G_{k l}^{G^{W}}\right), \\
0 & \text { otherwise, }
\end{array}\right.
$$

for $k, l=1, \ldots, K$, where $B R G_{k l}^{G^{W}}$ is the bearing between the $k$-th and $l$-th pattern landmarks.

The task of the identification system is to find in the set of pattern landmarks and in the set of landmarks of

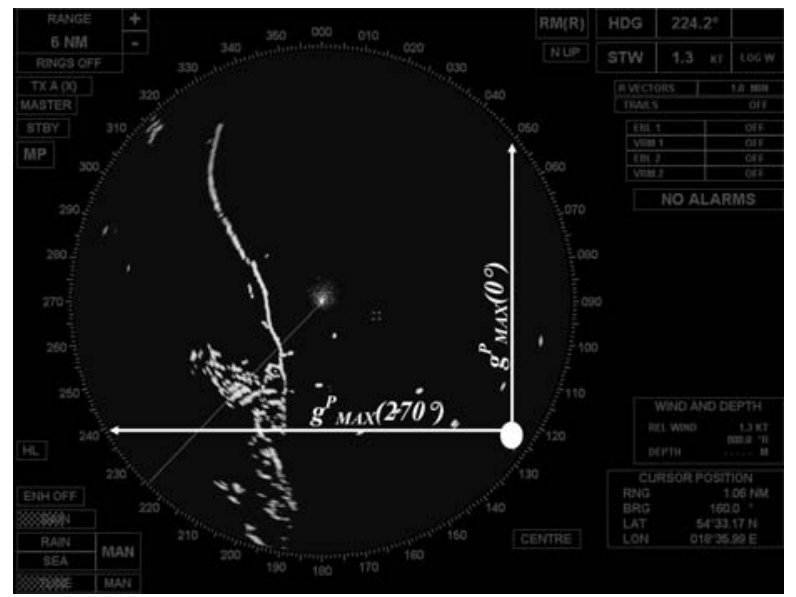

Fig. 3. Furthest points visible from a given radar image point for $B R G=0^{\circ}$ and $B R G=270^{\circ}$.

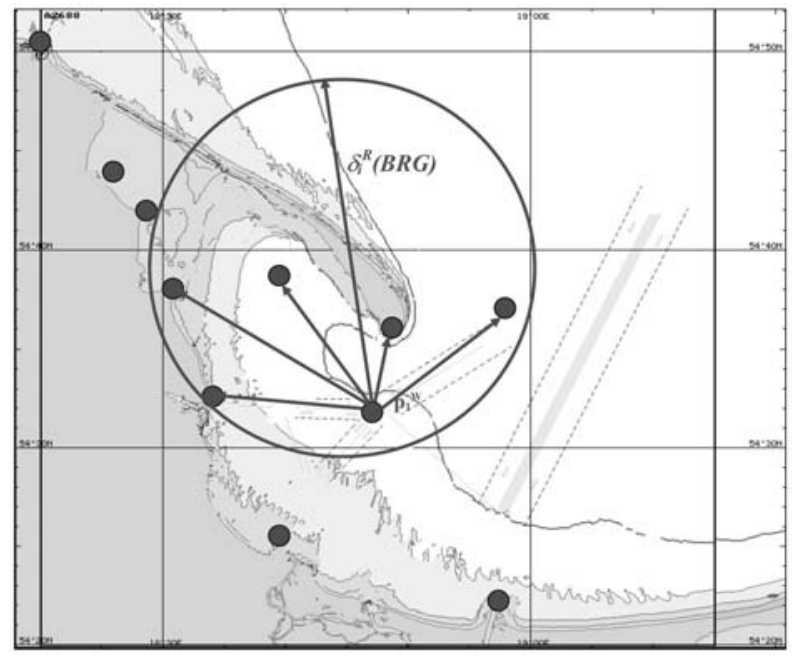

Fig. 4. Description of the point $p^{W}$.

the radar image two elements for which the corresponding trees match each other best. The matching of trees concerns their edges, i.e. the bearings and distances that describe every edge.

Take any two points $p_{i}^{W} \in P^{W}$ and $p_{j}^{I} \in P^{I}$. A tree corresponds to every such point. $G^{I, j}$ describes a point of the radar image and $G_{R}^{W, i, j}$ defines a pattern landmark. Assume that to every edge from $G^{I, j}$ there is assigned a unique number from the range $1, \ldots, M$ $\left(M=\left|P^{I}\right|\right)$. A similar procedure is applied in relation to edges from $G_{R}^{W, i, j}$. They have indices from the range $1, \ldots, N\left(N=\left|P_{R}^{W, i, j}\right|\right)$, where $P_{R}^{W, i, j}$ is the set of pattern landmarks present in $G_{R}^{W, i, j}$

Let us now represent the edges of either tree as vertices of the graph $G_{i j}^{D}$. $G_{i j}^{D}$ is constructed in such a way that the set of its vertices is divided into two disjoint subsets. The edges $g_{k}^{G^{I, j}}, k=1, \ldots, M$ from $G^{I, j}$ constitute 
the first subset and the edges $g_{l}^{G_{R}^{W, i, j}}, l=1, \ldots, N$ from $G_{R}^{W, i, j}$ belong to the second subset. The matrix of mutual edge assignments

$$
\boldsymbol{A}^{G_{i j}^{D}}=\left[a_{l k}^{G_{i j}^{D}}\right]_{N \times M}
$$

completes the definition of the graph $G_{i j}^{D}$. Here

$a_{l k}^{G_{i j}^{D}}= \begin{cases}1 & \text { there is a conection from } g_{l}^{G_{R}^{W, i, j}} \text { to } g_{k}^{G^{I, j}}, \\ 0 & \text { otherwise, }\end{cases}$ which fulfils the following condition:

$$
\text { If } \begin{array}{r}
a_{l k}^{G_{i j}^{D}}=1 \text { and } a_{p r}^{G_{i j}^{D}}=1 \text { then } l \neq p \text { and } k \neq r, \\
\forall l, p=1, \ldots, N \text { and } k, r=1, \ldots, M
\end{array}
$$

for any $i=1, \ldots, K$ and $j=1, \ldots, L$.

The condition (8) means that to every edge from $G^{I, j}$ we can assign at most one edge from $G_{R}^{W, i, j}$. The same concerns edges from $G_{R}^{W, i, j}$ to which we can assign maximally one edge from $G^{I, j}$.

The similarity between individual edges is described by the matrix $\boldsymbol{B}_{i j}=\left[b_{l k}^{i j}\right]_{N \times M}$, where

$$
\begin{aligned}
b_{l k}^{i j} & =\left|g_{l}^{G_{R}^{W, i, j}} g_{k}^{G^{I, j}}\right| \\
& =\sqrt{\left(B R G_{l}^{G_{R}^{W, i, j}}-B R G_{k}^{G^{I, j}}\right)^{2}+\left(d_{l}^{G_{R}^{W, i, j}}-d_{k}^{G^{I, j}}\right)^{2}} .
\end{aligned}
$$

Let us denote by $b_{\max }$ the maximal acceptable value for the matching of edges. Now, it is possible to reduce the number of valid assignments only to these assignments for which all components fulfil the condition below:

$$
a_{l k}^{G_{i j}^{D}}=1 \Leftrightarrow b_{l k}^{i j} \leq b_{\max } .
$$

Summarizing, the matrix $\boldsymbol{B}_{i j}$, the factor $b_{\max }$ and the condition (8) determine the set $\Omega_{\boldsymbol{B}_{i j}}^{b_{\max }}$ of acceptable graphs $G_{i j}^{D}$ that we can create for a given pair $\left(p_{i}^{W}, p_{j}^{I}\right)$.

4.2. Algorithm of Landmark Identification. The first part of the algorithm consists in finding, for each pair of points $\left(p_{i}^{W}, p_{j}^{I}\right)$, a graph that defines an edge assignment for which we are not able to find a better assignment. Denote by $G_{i j}^{D^{*}}$ the desired graph.

The evaluation of any graph $G^{D} \in \Omega_{B}^{b_{\max }}$ yields

$$
\begin{aligned}
\boldsymbol{E}^{1}\left(G^{D}\right) & =\left(Z\left(\boldsymbol{A}^{G^{D}}\right), S_{\boldsymbol{B}}\left(\boldsymbol{A}^{G^{D}}\right)\right) \\
Z\left(\boldsymbol{A}^{G^{D}}\right) & =\sum_{l}^{N} \sum_{k}^{M} a_{l k}^{G^{D}}, \\
S_{\boldsymbol{B}}\left(\boldsymbol{A}^{G^{D}}\right) & =\frac{1}{\sum_{l}^{N} \sum_{k}^{M} b_{l k} a_{l k}^{G^{D}}} .
\end{aligned}
$$

Here $Z\left(\boldsymbol{A}^{G^{D}}\right)$ informs us about the number of matched edges at the level determined by $b_{\max }$, whereas $S_{\boldsymbol{B}}\left(\boldsymbol{A}^{G^{D}}\right)$ determines the total matching degree of individual edges.

If we define the relation $\succeq \subset \mathbb{N} \times \mathbb{R}$ in the following way:

$$
(x, y) \succeq 2^{2}(z, r) \Leftrightarrow(x>z) \text { or } \quad(x=z \text { and } y \geq r),
$$

then the definition of the graph $G^{D^{*}}$ is

$$
\boldsymbol{E}^{1}\left(G^{D *}\right) \stackrel{2}{\succeq} \boldsymbol{E}^{1}\left(G^{D}\right), \quad \forall G^{D} \in \Omega_{\boldsymbol{B}}^{b_{\max }} .
$$

In practice, the situation in which more than one graph fulfils (15) is rather impossible. Theoretically, however, we cannot exclude it. In this case, we have to choose arbitrarily one graph which will represent the assignment of points in further calculations.

The second task of the algorithm is to find a pair of points $\left(p_{i}^{W}, p_{j}^{I}\right)^{*}$ that fulfils the following relation:

$$
\begin{aligned}
& \boldsymbol{E}^{2}\left(\left(p_{i}^{W}, p_{j}^{I}\right)^{*}\right) \succeq \boldsymbol{E}^{2}\left(\left(p_{k}^{W}, p_{l}^{I}\right)\right), \\
& \forall i, k=1, \ldots, K, \quad \forall j, l=1, \ldots, L,
\end{aligned}
$$

where $\stackrel{3}{\succeq}$ is the relation defined on $\mathbb{N} \times \mathbb{R} \times \mathbb{R}$ such that

$$
\begin{gathered}
(x, y, p) \succeq(z, r, s) \Leftrightarrow(x>z) \text { or }(x=z \text { and } y>r) \\
\text { or }(x=p \text { and } y=r \text { and } p \geq s) .
\end{gathered}
$$

$\boldsymbol{E}^{2}$ is defined as follows:

$$
\boldsymbol{E}^{2}\left(\left(p_{i}^{W}, p_{j}^{I}\right)\right)=\left(Z\left(\boldsymbol{A}^{G_{i j}^{D^{*}}}\right), S_{\boldsymbol{B}_{i j}}\left(\boldsymbol{A}^{G_{i j}^{D^{*}}}\right), \frac{1}{\left|p_{j}^{I} p_{c}\right|}\right) .
$$

The above procedure of selecting $\left(p^{W}, p^{I}\right)^{*}$ means that among all possible pairs we are most interested in a pair whose graph $G^{D^{*}}$ does not have a better equivalent among the graphs representing the remaining pairs. Another important factor during the selection of $\left(p^{W}, p^{I}\right)^{*}$ is the distance of the point $p^{I}$ to the central point of the radar image in which the ship is located. This distance is important for the accuracy of the estimated position. The point $p^{I}$ constitutes a basic reference point in the fixing position process so its correct location in the radar image is very important. Given that the position of any characteristic point in the radar image is determined with some error which is proportional to the distance to this point, it is crucial that the point whereby the position of the ship is estimated be located as close to our ship as possible.

The pair $\left(p_{i}^{W}, p_{j}^{I}\right)^{*}$ and the graph $G_{i j}^{D^{*}}$ allow us to determine the position of the point $p_{j}^{I}$ as well as other points considered in the matrix $A^{G_{i j}^{D^{*}}}$ and occurring in the graph $G^{I, j}$. Eventually, knowledge about the location of radar image points and the application of traditional methods of radar navigation yields a potential to estimate the ship's position. 


\section{Verification of the Algorithm}

The verification of the algorithm was performed in the following way: A pattern image of $100 \times 100$ pixels size was generated. Pattern points (60 or 20 points) imitating pattern landmarks were located in the pattern image. The distribution of the points was uniform. In the pattern image, test points were also located that imitated the ship's position from which radar observations were conducted (81 evenly distributed points). Then, for each test point, points that were noisy equivalents of the pattern landmarks, observed from the test position, for the radar observation range of 24 or 12 pixels (the size of the observed area) were generated. The direction of the introduced noise was random while its value depended on the distance of a given pattern point to the test point $( \pm 30 \%, \pm 20 \%$ and $\pm 10 \%$ of the distance between the points). Furthermore, to simulate the possibility of generating by the landmark extraction subsystem additional points that do not occur in the database of pattern landmarks, points at random location were introduced to the set of noisy pattern points for each test point. The number of additional points was dependent on the number of noisy pattern points observed from the given test point ( $10 \%$ or $30 \%$ of this number). During the experiments, four values of $b_{\max }$ were assumed: 2, 5, 10 and 30.

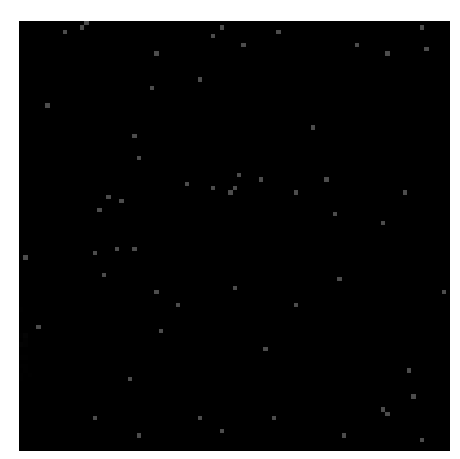

Fig. 5. Sample deployment of pattern points.

The tests conducted consisted in fixing the pair $\left(p^{W}, p^{I}\right)^{*}$ in each out of all test points ( $p^{W}$ denotes one of pattern points whereas $p^{I}$ is one of the noisy pattern points visible from the test point). The following results produced by the algorithm were possible: a proper point assignment, a wrong assignment or a situation in which the algorithm was not able to identify any characteristic point of the radar image (a noisy pattern point). The latter could happen in the case when the value of $b_{\max }$ was too demanding and for every landmarks of the radar image the algorithm could not find a sufficiently good equivalent among pattern points. Below, sample results of the experiments are presented.

As the tests conducted have exhibited, the distribution of the pattern landmarks has larger influence on the algorithm performance than the occurrence of additional points. The algorithm is able to eliminate the additional characteristic points generated by the landmark extraction subsystem. If the additional points do not dominate in the set of points observed in the radar image, then they should not significantly affect the identification results.

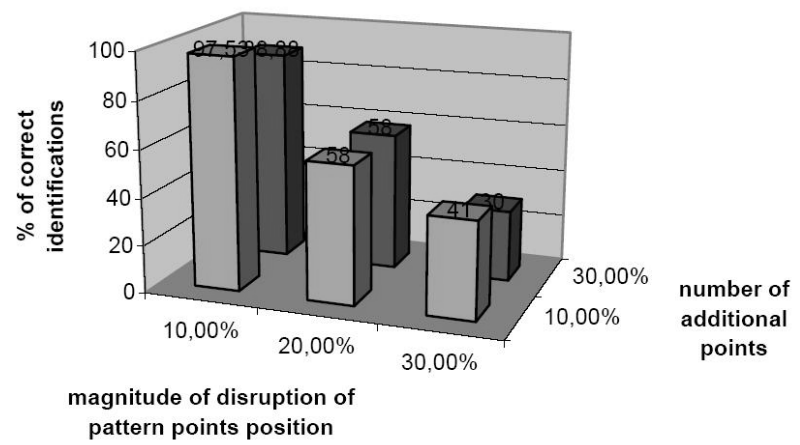

Fig. 6. Influence of the number of additionally generated characteristic points and the magnitude of displacement of noisy pattern points with respect to the original pattern points on landmarks identification (the number of pattern points is $60, b_{\max }=2, R=24$ ).

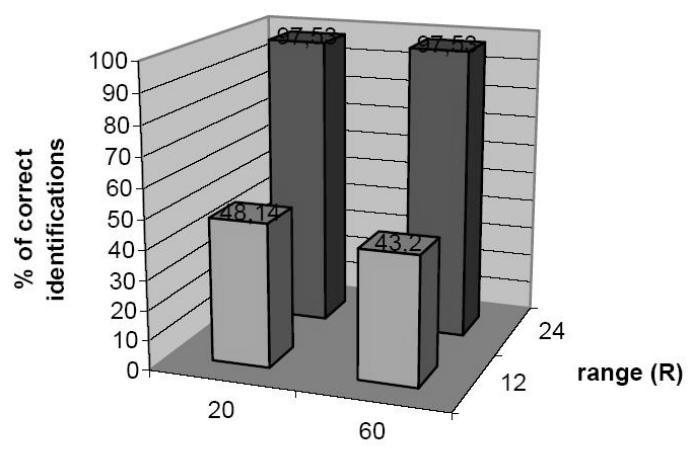

number of pattern points

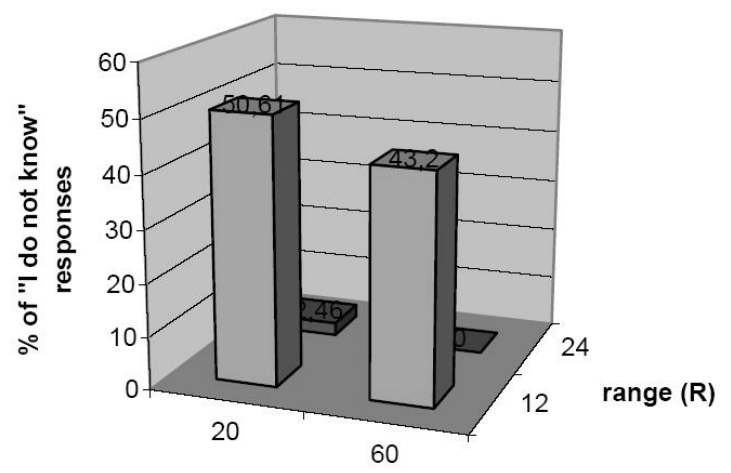

number of pattern points

Fig. 7. Influence of the number of pattern points memorized in the database and the range of radar observations $(R)$ on the identification results (the number of additional points is equal to $10 \%$ of pattern points visible from the test point, the magnitude of displacement of pattern points is equal to $10 \%$ of the distance to the test point, $b_{\max }=2$ ). 
Moreover, the tests revealed that the number of simultaneously observed landmarks has stronger influence on the algorithm performance. For larger $R$ more characteristic points are visible and the algorithm is highly effective. It is so even though points further located, visible only for greater values of $R$, get more and more noisy. In the case of the application of a shorter range, the percentage of points correctly identified drastically decreases, causing at the same time a rapid growth in the "I do not know" responses.

Figure 8 illustrates once again large influence of the radar observation range on the obtained results. They also show that the parameter $b_{\max }$ influences the gradual increase in the number of "I do not know" responses in the case of the application of a short radar observation range. Its impact on the percentage of correct identifications is not significant.
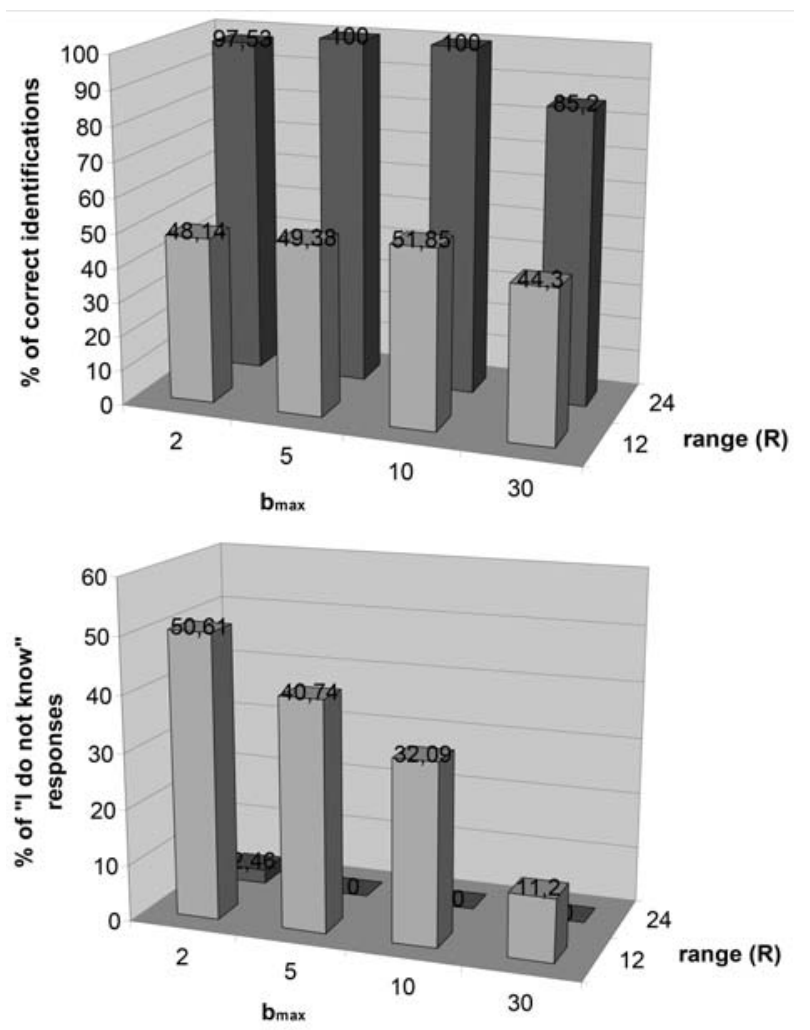

Fig. 8. Influence of $b_{\max }$ and $R$ on the algorithm performance (the number of additional pattern points equals $10 \%$ of the points visible from the test point, the magnitude of displacement of pattern points equals $10 \%$ of the distance to the test point, the number of pattern points is 20).

\section{Conclusions}

An identification algorithm of radar image characteristic points has been presented. The proposed approach is based on the comparison of pattern bearings and distance trees, fixed for pattern points (from a chart) and bearing and distance trees representing points extracted from a radar image. The tests conducted exhibited a potential of the algorithm to perform the identification task. They also revealed that the radar observation range and the distribution of characteristic points observed with respect to their pattern positions have the greatest influence on the algorithm performance. A very important feature of the algorithm is its robustness to the presence, among visible characteristic points, of additional points absent in the pattern point database and generated by the landmark extraction subsystem. Moreover, it is worth remembering that the decisions made by the presented identification system can be verified by another identification system, e.g. by the landmark identification system that makes decisions based on the shape of the coast visible from a given pattern point (Praczyk, 2006a). Both systems would complement each other. They can, e.g. generate an ordered sequence of pairs of points (a pattern point, a point appearing in the radar image), from the best matched pair to the worst matched one. The final decision, i.e. selecting the best pair, would be taken based on the location of every pair in both of the ordered lists. The pair whose total distance from the beginning of each list would be the least would constitute the basis in further calculations associated with estimating the ship's position.

An interesting issue demanding further research is the possibility of applying bearing and distance trees to estimate the most appropriate location for landmarks in the radar image. Usually every characteristic point present in the radar image is solely an approximation of the true pattern point. Hence, the position estimated based on it is frequently inaccurate. However, if we only could move a radar image point to a more suitable place in the image, the estimated position would be more accurate. In order to achieve this result, it is perhaps necessary to move a given point in different directions to adjust its tree to the pattern tree.

\section{References}

Kuchariew G. (1999): Processing and Digital Images Analysis. — Szczecin: Technical University Press, (in Polish).

Praczyk T. (2006a): Automatic radar navigational system. Theoretical and Applied Informatics, Vol. 18, No. 2, pp. 91-108.

Praczyk T. (2006b): Artificial neural networks application in maritime, coastal, spare positioning system. - Theoretical and Applied Informatics, Vol. 18, No. 3, pp. 175-188.

Praczyk T. (2006c): Application of neural networks and radar navigational aids of shore area to positioning. - Computational Methods in Science and Technology, Vol. 12, No. 2, pp. 53-59. 
Praczyk T. (2006d): Better Kohonen neural network in radar images compression. - Computational Methods in Science and Technology, Vo. 12, No. 2, pp. 61-68.
Stateczny A. (2001): Comparative Navigation. - Gdańsk: Gdańsk Scientific Society, (in Polish).

Received: 6 June 2006

Revised: 27 December 2006 